\title{
Elimination of onchocerciasis in Africa by 2025: the need for a broad perspective
}

\author{
Ed Cupp ${ }^{1}$, Mauricio Sauerbrey ${ }^{2}$, Vitaliano Cama ${ }^{3}$, Mark Eberhard ${ }^{3}$, Patrick J. Lammie ${ }^{4}$ and Thomas R. Unnasch ${ }^{5^{*}}$ (D
}

\begin{abstract}
Background: In response to the recent publication "Is onchocerciasis elimination in Africa feasible by 2025: a perspective based on lessons learnt from the African control programmes" by Dadzie et al., it is important to clarify and highlight the positive and unequivocal research and operational contributions from the American experience towards the worldwide elimination of human onchocerciasis (river blindness).

Main text: The strategies of twice or more rounds of mass drug administration (MDA) of ivermectin per year, as well as the use of OV-16 serology have allowed four American countries to be verified by World Health Organization to have eliminated transmission of Onchocerca volvulus, the etiological agent. These advances were also implemented in Sudan and Uganda; currently, both are the only African countries where ivermectin MDA was safely stopped in several transmission zones.
\end{abstract}

Conclusions: Programmatic treatment and evaluation approaches, pioneered in the Americas, are the most efficient among the existing tools for elimination, and their broader use could catalyze the successful elimination of this disease in Africa.

Keywords: Onchocerciasis, Ivermectin, Onchocerciasis in the Americas, Elimination, African Programme for onchocerciasis control, Onchocerciasis control program of West Africa

\section{Multilingual abstracts}

Please see Additional file 1 for translations of the abstract into the six official working languages of the United Nations.

\section{Background}

The recent publication "Is onchocerciasis elimination in Africa feasible by 2025: a perspective based on lessons learnt from the African control programmes" by Dadzie et al. [1] is quite informative in recounting the evolution of onchocerciasis control programs in Africa and the history behind the decision to move from control to elimination. As pointed out by Dadzie et al. [1], onchocerciasis has long been the focus of the international community, due to its associated morbidities, including blindness, skin disease and an association with

\footnotetext{
* Correspondence: tunnasch@health.usf.edu

Mark Eberhard has retired

${ }^{5}$ Center for Global Health Infectious Diseases, University of South Florida College of Public Health, 3720 Spectrum Blvd, Suite 304, Tampa, FL 33612, USA

Full list of author information is available at the end of the article
}

childhood epilepsy [2]. However, in several instances, Dadzie et al. mischaracterizes the contributions that the regional initiative to eliminate onchocerciasis in the Americas (OEPA) has made to the broader effort in Africa. We believe that key findings of OEPA's experience have either been overlooked by the onchocerciasis control and elimination programs in Africa or the nuances of the situation in Latin America were not completely understood by policy makers. Further, Dadzie et al. express concern that Africa has been unduly "influenced by the relatively limited American experience." Here, we focus on several key examples raised in that article and offer comment based on decades of OEPA's experience and examples from the published, refereed literature, which we feel could inform and guide the African programs. A serious effort to eliminate onchocerciasis was started in by OEPA 1993. 25 years later, four American countries have been verified by World Health Organization (WHO) to have successfully interrupted the transmission of Onchocerca volvulus, the causative agent of onchocerciasis. Thus, we believe there are important contributions from the American experience

(c) The Author(s). 2019 Open Access This article is distributed under the terms of the Creative Commons Attribution 4.0 International License (http://creativecommons.org/licenses/by/4.0/), which permits unrestricted use, distribution, and 
that could benefit many African countries. Indeed, the American experience with onchocerciasis has played a leading role in the study of this disease throughout history. The connection between onchocerciasis and ocular disease was first discovered in the Americas, by the Guatemalan investigator Dr. Rodolfo Robles in 1919 [3], followed later by the link between onchocerciasis and epilepsy reported by Dr. Casis-Sacre in Mexico in 1938 [4]. These seminal clinical findings re-enforce the region's historical, long-standing focus on this important disease of poverty.

\section{Main text}

\section{The use of twice per year mass drug administration (MDA) of ivermectin}

OEPA pioneered the use of twice per year treatments at high coverage rates, and in so doing has eliminated onchocerciasis from four of the six countries affected in the region; Colombia [5], Ecuador [6], Mexico [7] and Guatemala [8]. This scale up strategy in the American region from once to twice per year MDA began around 2000, based on a series of earlier studies first in Africa [9-12] and a 3 year pilot study in Guatemala [13] confirming the effectiveness of this regimen. These African and American studies formed the basis for the OEPA strategy of transmission elimination using twice per year ivermectin treatment. Consequently, more than 500000 people no longer need ivermectin in the Americas [14] and children born in those 11 formerly endemic foci over the past decade are free of the risk of onchocerciasis and its associated pathologies. This effort represents a significant contribution towards the prevention of blindness globally. Using similar strategies and metrics, even greater numbers of individuals from Sudan and Uganda have been freed from the risk of onchocerciasis-related skin and eye disease as well.

It should be noted that the Onchocerciasis Control Program of West Africa (OCP) was also using a strategy of administration of ivermectin twice per year in many areas at the same time OEPA was doing so [15]. While OCP's core activity was vector control, it had the same end goal as OEPA - interrupting parasite transmission. The main difference between these programs and the African Programme for Onchocerciasis Control (APOC) was that OEPA consistently chose to deploy twice per year ivermectin treatments in all endemic communities. In contrast, APOC sought to achieve sustained morbidity control by targeting only communities with the highest infection rates $(>20 \%$ nodule prevalence in adult males). The APOC strategy to control onchocerciasis as a public health problem was predicated on community directed annual treatments. APOC continued to advocate this annual treatment strategy even after the goal shifted to elimination of transmission rather than control. Thus, as reported in the final external review of the program [16], APOC was not successful in making a transition from a morbidity control program to a transmission elimination program. Outside of research and settings where vector control has historically been implemented by OCP, APOC never stopped treatments in an area where annual treatment was the primary intervention employed.

In December 31, 2015, APOC published a report describing strategic options and alternative treatment strategies for accelerating the elimination of onchocerciasis in Africa, and proposed the use of twice per year MDA, a core strategy developed and implemented by OEPA [17]. As noted above, two African countries (Sudan and Uganda) had already embraced the OEPA strategy [18-20] and demonstrated the elimination of transmission of $O$. volvulus in at least two endemic zones. Indeed, we contend that the twice per year MDA concept and the successes from the Americas became a foundational experience that moved those African programs from a control to an elimination paradigm.

\section{Stop MDA assessments based on serological testing rather than reliance on skin snip microscopy}

Another long-standing OEPA strategy included serological testing and exclusion of skin snip microscopy in its assessments to determine the safe stoppage of MDA. This approach was incorporated into the recent WHO Guidelines for Stopping Mass Drug Administration and Verifying Elimination of Human Onchocerciasis: Criteria and Procedures (Guidelines) [21], which were developed by the Guidelines Development Group, composed of experts in onchocerciasis. To ensure intellectual rigor, these recommendations were also vetted through a systematic review process conducted by independent Grading of Recommendations Assessment, Development and Evaluation (GRADE) methodologists at WHO. Therefore, the recommendations in the 2016 Guidelines were systematically reviewed at two levels. Both skin snip microscopy and serology were determined to have "low certainty of evidence" in this process; however, the serological assay was given a "strong recommendation" while skin snip microscopy was given a "conditional recommendation". Since the publication of the 2016 Guidelines, there have been additional reports comparing the performance of skin snip microscopy to polymerase chain reaction (PCR) amplification of parasite DNA from skin snips [22, 23], showing that skin snip microscopy significantly under-detects infections, with more pronounced deficits when $O$. volvulus microfilariae loads were low, which is the situation that programs encounter in hypo-endemic areas and in areas under successful MDA. These recent peer-reviewed reports reinforce the 2016 Guidelines recommendation that skin snip microscopy is not adequate to demonstrate the interruption of transmission of onchocerciasis. The current OV-16 
serological assay is not perfect; it has to be used within very specific age range and employ statistically meaningful sample sizes to be informative. However, when used as recommended, it reflects the cumulative incidence of infection in the sampled population, and in conjunction with entomology helps determine whether transmission of $O$. volvulus has been interrupted. For the latter, the prevalence of black flies carrying infective larvae $\left(\mathrm{L}_{3} \mathrm{~s}\right)$ in the head of less than $0.1 \%$ in parous flies or less than $0.05 \%$ in all flies (assuming a parity rate of $50 \%$ ) are criteria used for entomological assessments. While the entomological metrics are difficult and expensive to achieve using conventional techniques, molecular methods (e.g. pool screen PCR [24]) and recently reported community directed methods for collecting vectors that can replace human landing collections [25] have made achieving the metrics set out in the guidelines both practical and affordable.

\section{Ivermectin as an $O$. volvulus macrofilaricide}

Dr. Brian Duke and others working in the Americas pioneered the concept that repeated ivermectin treatments (twice or 4 times a year) were macrofilaricidal and that the impact of ivermectin on adult O. volvulus was best observed under conditions in which parasite transmission had been interrupted [26, 27]. Further, the long-term operational effect of repetitive twice/year treatments on adult worm survival and mating in the Americas showed that semi-annual treatments over 6-7 years at high coverage rates were generally equivalent to $10-13$ years of vector control. This information was reported in 2004 [28]. Unfortunately, these seminal American research publications did not receive the attention they perhaps deserved by the African programs, leading some to unfairly conclude that the American experience was "relatively limited."

\section{Epidemiological models}

Dadzie et al. highlight the fact that APOC relied heavily on models to inform their strategy. In fact, both OCP and APOC worked closely with modelers to develop predictions for the effects of vector control and ivermectin MDA on the dynamics of infection in the human population and transmission of $O$. volvulus. This collaboration resulted in the development of at least three different models for onchocerciasis predictions: The Computer Simulation Program for Transmission and Control of Onchocerciasis (ONCHOSIM), EpiOncho and the Simulation Model of Onchocerciasis (SIMON) [29, 30]. For the Americas, OEPA adapted one of these models (SIMON) for use in Latin America as early as 2003, designating this modified model SIMONA, or Simulation Model of Onchocerciasis in the Americas [31]. Thus, the American and African programs both attempted to develop and utilize models to inform their decisions. Of course, the value of relying on models to make such strategic decisions is predicated on the underlying accuracy of the model predictions. In this regard, it is disturbing that the two models developed with APOC and OCP support (EPIONCHO and ONCHOSIM) have produced predictions that varied widely in some operationally important areas, such as the risk of recrudescence of infection following cessation of annual ivermectin distribution in areas where vector biting rates are high [32]. Additional work is needed to determine why this is the case, and to attempt to harmonize the results obtained by the models. Until this is done, it is difficult to determine which of these African prediction models should be used to inform important programmatic decisions, such as when it is safe to discontinue treatments. Furthermore, in the only operational test of the validity of the predictions of the models and epidemiological criteria relied upon by APOC, transmission was found to be ongoing in $3 / 5$ countries where APOC's criteria had indicated elimination had been achieved [33]. These findings suggest that the methods and metrics developed by APOC may inaccurately predict programmatic success at a high frequency.

\section{Elimination operational algorithms}

OEPA's original objective was to reach a point when ivermectin treatment could be successfully and safely withdrawn. Given the encouraging progress in three of the six endemic countries at the time, the annual InterAmerican Conference on Onchocerciasis (IACO) in 1996 concluded that the development of internationally accepted standards for certification of onchocerciasis transmission elimination were urgently needed [34]. [NB: WHO originally used the term "certification" to indicate institutional approval but has since chosen to use "verification" in its place.] OEPA's steering committee (the Program Coordinating Committee, PCC) began to draft these criteria in 1997. This work included the conceptual control/elimination algorithm recently cited in Dadzie et al. [1]. During the same period, President Jimmy Carter pressed then WHO Director General Gro Harlem Brundtland for leadership on this issue. WHO headquarters rejected the idea of having elimination guidelines that were only applicable to the Americas Region, and convened a consultative meeting in Geneva in 2000 to develop global guidelines that involved experts from OCP, OEPA, and APOC. The baseline document for this meeting was written by OEPA PCC members Ed Cupp, Richard Collins, and Frank Richards. The OEPA based algorithms later appeared in the first WHO Guidelines for Certification of Elimination of $\mathrm{Hu}$ man Onchocerciasis: Criteria and Procedures [35]. Dadzie et al. [1] chose to adopt this algorithm for both the OCP (\# 1) and APOC (\# 3) figures, endorsing, perhaps unknowingly, OEPA's seminal role in this process. APOC rarely if ever 
cited the $2001 \mathrm{WHO}$ onchocerciasis elimination document, despite the fact that it was officially endorsed by $\mathrm{WHO}$ as a global strategy and published by WHO in English, French, Portuguese and Spanish.

\section{Vectorial capacity}

When compared to much of Africa, onchocerciasis in the Americas was generally of lower intensity. However, many American foci were hyperendemic for the disease because of the extremely high annual biting rates by less efficient vector black flies due to their cibarial armature (Simulium ochraceum in Guatemala and Mexico, for example). However, S. exiguum in Ecuador has an equivalent vectorial capacity to savannah $S$. damnosum species [36], and hyperendemic communities in Ecuador had baseline community prevalences of $>90 \%$, a situation very similar to that seen in the most hyper-endemic African settings. Yet $O$. volvulus transmission was eliminated in Ecuador after 9 years of twice per year treatment [6]. Around the same time as MDA was halted in Ecuador, the 2009 Diawara study in Mali and Senegal proclaimed the proof of principle of elimination of onchocerciasis in Africa with ivermectin mass treatment alone [15]. Unfortunately, Diawara et al. were unable to determine whether twice per year treatment could accelerate the process to under 15 years [15]. The Ecuador results would have helped shed light on this, but the relevancy of the success in Ecuador was not fully appreciated by the APOC community. Indeed, the Mali/ Senegal report (which soon gave way to APOC elimination guidelines) also made no mention of the official 2001 WHO published guidelines, and especially ignored the principle of monitoring infection in children as a measure of recent incidence.

\section{Conclusions}

OEPA's experience, and its final success (or failure) will continue to provide lessons of the end game that should be appreciated by current African programs as their quest for transmission elimination continues. The American programs are now challenged with eliminating transmission in all areas, and strengthening both posttreatment and post-elimination surveillance. For example, OEPA is now engaged in the use of doxycycline and 4 times per year ivermectin treatment as new tools to assure finality of elimination. Some African programs will soon reach the point where OEPA is now, having stopped $94 \%$ of its treatments. Thus, African program managers ought to look carefully at the OEPA experience to discover and learn from past successes and potentially pertinent new approaches to dealing with nontechnical issues of population migration, political instability, insecurity, cross border challenges, and waning political and financial support that are so fundamental to address in order to finish the job. We urge them to do so.

\section{Additional file}

Additional file 1 Multilingual abstracts in the six official working languages of the United Nations. (PDF 291 kb)

\section{Abbreviations}

APOC: African Programme for Onchocerciasis Control; GRADE: Grading of Recommendations Assessment, Development and Evaluation; IACO: InterAmerican Conference on Onchocerciasis; MDA: Mass drug administration of ivermectin; OCP: Onchocerciasis Control Programme; OEPA: Onchocerciasis Elimination Program for the Americas; PCC: Program Coordinating Committee; WHO: World Health Organization

\section{Acknowledgements}

We would like to thank Frank Richards of the Carter Center for critically reading the manuscript.

\section{Disclaimer}

The findings and conclusions in this report are those of the authors and do not necessarily represent the views of the Centers for Disease Control and Prevention.

\section{Authors' contributions}

EC, MS, VC, ME, PL and TRU contributed to the writing, reviewing and revision of the manuscript. All authors read and approved the final manuscript.

\section{Authors' information}

MS is the director of the Carter Center Onchocerciasis Elimination Program for the Americas. VC is a microbiologist in the Division of Parasitic Diseases and Malaria at the Centers for Diseases Control and Prevention. ME is the former director of the Division of Parasitic Diseases and Malaria at the Centers for Diseases Control and Prevention. PL is the Chief Scientist of the Neglected Tropical Diseases Support Center of the Task Force for Global Health. TRU is the Chair of the Onchocerciasis Technical Subcommittee of the World Health Organization, the Chair of the Uganda Onchocerciasis Elimination Expert Advisory Committee and the Director of the World Health Organization Collaborating Centre for Onchocerciasis Diagnostics. EC, PL and TRU were members of the World Health Organization Guidelines Development Group responsible for writing the Guidelines for Stopping Mass Drug Administration and Verifying Elimination of Human Onchocerciasis. ME was the chair of the Guidelines Development Group. All authors read and approved the final manuscript.

Funding

Not applicable.

Availability of data and materials Not applicable.

Ethics approval and consent to participate Not applicable.

\section{Consent for publication}

Not applicable.

\section{Competing interests}

The authors declare that they have no financial or non-financial competing interests.

\section{Author details}

'Department of Entomology and Plant Pathology, Auburn University, Auburn, AL 36849, USA. ${ }^{2}$ Onchocerciasis Elimination Program for the Americas, 14 Calle 3-51 Zona 10, Edificio Murano Center, Oficina, 1401 Guatemala City, Guatemala. ${ }^{3}$ Centers for Disease Control and Prevention, 1600 Clifton Rd, MS D-65, Atlanta, GA 30329, USA. ${ }^{4}$ Task Force for Global 
Health, 330 W. Ponce de Leon Ave, Decatur, GA 30030, USA. ${ }^{5}$ Center for Global Health Infectious Diseases, University of South Florida College of Public Health, 3720 Spectrum Blvd, Suite 304, Tampa, FL 33612, USA.

Received: 26 November 2018 Accepted: 28 May 2019

Published online: 15 July 2019

\section{References}

1. Dadzie Y, Amazigo UV, Boatin BA, Seketeli A. Is onchocerciasis elimination in Africa feasible by 2025: a perspective based on lessons learnt from the African control programmes. Inf Dis Poverty. 2018;7(1):63.

2. Colebunders R, J YC, Olore PC, Puok K, Bhattacharyya S, Menon S, et al. High prevalence of onchocerciasis-associated epilepsy in villages in Maridi County, republic of South Sudan: a community-based survey. Seizure. 2018;63:93-101.

3. Robles R. Onchocercose hujaine au Guatémala produisant le cécité el " l'érysipele du litoral" (Erisipela de la costa). Bull Soc Pathol Exot. 1919;7:442-63. (in French).

4. Casis-Sacre G. El syndrome epiléptico y sus relaciones con la oncocercosis. Bol Salubre Hig (Mexico). 1938;1:11-31.

5. Nicholls RS, Duque S, Olaya LA, Lopez MC, Sanchez SB, Morales AL, et al. Elimination of onchocerciasis from Colombia: first proof of concept of river blindness elimination in the world. Parasit Vectors. 2018;11(1):237.

6. Guevara A, Lovato R, Proano R, Rodriguez-Perez MA, Unnasch T, Cooper PJ, et al. Elimination of onchocerciasis in Ecuador: findings of post-treatment surveillance. Parasit Vectors. 2018:11(1):265.

7. Rodriguez-Perez MA, Fernandez-Santos NA, Orozco-Algarra ME, RodriguezAtanacio JA, Dominguez-Vazquez A, Rodriguez-Morales KB, et al. Elimination of onchocerciasis from Mexico. PLoS Negl Trop Dis. 2015;9(7):e0003922.

8. Anonymous. Progress towards eliminating onchocerciasis in the WHO region of the Americas: verification of elimination of transmission in Guatemala. Wkly Epidemiol Rec. 2016;91(43):501-5.

9. Taylor HR, Semba RD, Newland HS, Keyvan-Larijani E, White A, Dukuly Z, et al. Ivermectin treatment of patients with severe ocular onchocerciasis. Am J Trop Med Hyg. 1989:40(5):494-500.

10. Van der Lelij A, Rothova A, Klaassen-Broekema N, Wilson WR, Barbe RF, Stilma JS. Decrease in adverse reactions after repeated ivermectin treatment in onchocerciasis. Doc Ophthalmol. 1990;75(3-4):215-24.

11. Cupp EW, Bernardo MJ, Kiszewski AE, Collins RC, Taylor HR, Aziz MA, et al. The effects of ivermectin on transmission of Onchocerca volvulus. Science. 1986;231(4739):740-2.

12. Taylor HR, Pacque M, Munoz B, Greene BM. Impact of mass treatment of onchocerciasis with ivermectin on the transmission of infection. Science. 1990;250:116-8

13. Cupp EW, Ochoa JO, Collins RC, Cupp MS, Gonzales-Peralta C, Castro J, et al. The effects of repetitive community-wide ivermectin treatment on transmission of Onchocerca volvulus in Guatemala. Am J Trop Med Hyg. 1992;47:170-80.

14. World Health Organization. Onchocerciasis: Key facts WHO, Geneva 2018 Accessed 1 February, 2019 [updated 4 April 2018] http://www.who.int/ news-room/fact-sheets/detail/onchocerciasis

15. Diawara L, Traore MO, Badji A, Bissan Y, Doumbia K, Goita SF, et al. Feasibility of onchocerciasis elimination with ivermectin treatment in endemic foci in Africa: first evidence from studies in Mali and Senegal. PLoS Negl Trop Dis. 2009;3(7):e497.

16. Anonymous The WHO African Programme For Onchocerciasis Control. Final Evaluation Report. Ouagadougou, Burkina Faso: WHO/APOC; 2015. Document JAF21.6.

17. Sauerbrey $M$. The onchocerciasis elimination program for the Americas (OEPA). Ann Trop Med Parasitol. 2008;102(Suppl 1):25-9.

18. Higazi TB, Zarroug IMA, Mohamed HA, ElMubark WA, Deran TCM, Aziz N, et al. Interruption of Onchocerca volvulus transmission in the Abu Hamed focus, Sudan. Am J Trop Med Hyg. 2013;89(1):51-7.

19. Zarroug IM, Hashim K, ElMubark WA, Shumo ZA, Salih KA, ElNojomi NA, et al. The first confirmed elimination of an onchocerciasis focus in Africa: Abu Hamed, Sudan. Am J Trop Med Hyg. 2016;27:1037-40.

20. Lakwo T, Garms R, Wamani J, Tukahebwa EM, Byamukama E, Onapa AW, et al. Interruption of the transmission of Onchocerca volvulus in the Kashoya-Kitomi focus, western Uganda by long-term ivermectin treatment and elimination of the vector Simulium neavei by larviciding. Acta Trop. 2017;167:128-36.

21. World Health Organization. Onchocerciasis: Guidelines for stopping mass drug administration and verifying elimination of human onchocerciasis. Geneva: World Health Organization press; 2016. Document WHO/HTM/NTD/ PCT/2016.1.
22. Thiele EA, Cama VA, Lakwo T, Mekasha S, Abanyie F, Sleshi M, et al. Detection of Onchocerca volvulus in skin snips by microscopy and real-time polymerase chain reaction: implications for monitoring and evaluation activities. Am J Trop Med Hyg. 2016;94(4):906-11.

23. Prince-Guerra JL, Cama VA, Wilson N, Thiele EA, Likwela J, Ndakala N, et al. Comparison of PCR methods for Onchocerca volvulus detection in skin snip biopsies from the Tshopo Province, Democratic Republic of the Congo. Am J Trop Med Hyg. 2018;98(5):1427-34.

24. Katholi CR, Toe L, Merriweather A, Unnasch TR. Determining the prevalence of Onchocerca volvulus infection in vector populations by polymerase chain reaction screening of pools of black flies. J Infect Dis. 1995;172(5):1414-7.

25. Loum D, Katholi CR, Lakwo T, Habomugisha P, Tukahebwa EM, Unnasch TR. Evaluation of community-directed operation of black Fly traps for entomological surveillance of Onchocerca volvulus transmission in the Madimid north focus of onchocerciasis in northern Uganda. Am J Trop Med Hyg. 2017;97(4):1235-42.

26. Duke BO, Zea-Flores G, Castro J, Cupp EW, Munoz B. Comparison of the effects of a single dose and of four six-monthly doses of ivermectin on adult Onchocerca volvulus. Am J Trop Med Hyg. 1991;45(1):132-7.

27. Duke BO, Zea-Flores G, Castro J, Cupp EW, Munoz B. Effects of three-month doses of ivermectin on adult Onchocerca volvulus. Am J Trop Med Hyg. 1992:46(2):189-94

28. Cupp EW, Duke BO, Mackenzie CD, Guzman JR, Vieira JC, Mendez-Galvan J, et al. The effects of long-term community level treatment with ivermectin (Mectizan) on adult Onchocerca volvulus in Latin America. Am J Trop Med Hyg. 2004;71(5):602-7.

29. Basanez MG, Walker M, Turner HC, Coffeng LE, de Vlas SJ, Stolk WA. River Blindness: Mathematical Models for Control and Elimination. Adv Parasitol. 2016;94:247-341.

30. Davies JB. Description of a computer model of forest onchocerciasis transmission and its application to field scenarios of vector control and chemotherapy. Ann Trop Med Parasitol. 1993;87(1):41-63.

31. Dadzie $Y$, Neira M, Hopkins D. Final report of the conference on the eradicability of onchocerciasis. Filaria J. 2003;2(1):2.

32. Walker M, Stolk WA, Dixon MA, Bottomley C, Diawara L, Traore MO, et al. Modelling the elimination of river blindness using long-term epidemiological and programmatic data from Mali and Senegal. Epidemics. 2017;18:4-15.

33. Boakye D, Tallant J, Adjami A, Moussa S, Tekle A, Robalo M, et al. Refocusing vector assessment towards the elimination of onchocerciasis from Africa: a review of the current status in selected countries. Int Health. 2018;10(suppl_1):i27-32.

34. Anonymous. Onchocerciasis. Report from the InterAmerican Conference on Onchocerciasis in Oaxaca. Mexico Wkly Epidemiol Rec. 1997;72(29):215-8.

35. World Health Organization. Certification of elimination of human onchocerciasis : criteria and procedures. Geneva: World Health Organization; 2001. Report No.: WHO/CDS/CPE/CEE/2001.18b.

36. Collins RC, Lehmann T, Vieira Garcia JC, Guderian RH. Vector competence of Simulium exiguum for Onchocerca volvulus: implications for the epidemiology of onchocerciasis. Am J Trop Med Hyg. 1995;52:213-8.

\section{Ready to submit your research? Choose BMC and benefit from:}

- fast, convenient online submission

- thorough peer review by experienced researchers in your field

- rapid publication on acceptance

- support for research data, including large and complex data types

- gold Open Access which fosters wider collaboration and increased citations

- maximum visibility for your research: over $100 \mathrm{M}$ website views per year

At $\mathrm{BMC}$, research is always in progress.

Learn more biomedcentral.com/submission 